\title{
Characterization of a plane on a coordinate MEASURING MACHINE OF LARGE DIMENSIONS
}

Jaramillo, N. A.', Sánchez-Rinza, B. E. ${ }^{2}$

${ }^{1}$ Instituto Nacional de Astrofísica, Optica y Electrónica

Apdo. Post. \# 51 Y 216, 72000 Puebla, Pue., México.

${ }^{2}$ F. C. C., Benemérita Universidad Autónoma de Puebla Apdo. Post. \# 588,

72000 Puebla, Pue., México.

aramirs@siu.buap.mx, brinza@cs.buap.mx

Received: December 10 $0^{\text {th }}$, 2003. Accepted: September $9^{\text {th }}, 2005$

ABSTRACT

A technique for characterizing a horizontal plane within the measuring volume of a coordinate measuring machina (CMM) of large dimensions is described. The objective of the characterization is to obtain a correction table that allows us to minimize the mechanical errors introduced by its mechanical imperfection. The process contemplates the measurement of $Y$-axis yaw error and the squareness error between the $X$ and $Y$ axes of the machine, as well as obtaining a plane beginning with the characterization of a group of lines. This plane was then used to construct a correction table so that the uncertainty of measurement along the Z axis of the CMM could be reduced to less than 15 micrometers for any measurement carried out within an area of 4X5 m. In addition, a novel method for measuring the deviation angle of a pentaprism using the facilities of the CMM is described.

\section{RESUMEN}

En éste trabajo se describe un método empleado para caracterizar un plano horizontal dentro del volumen de medición de una máquina de medición por coordenadas de grandes dimensiones, con el propósito de construir una tabla de corrección que permitiera reducir los errores de la máquina introducidos por su imperfección mecánica y de ésta manera reducir la incertidumbre de medición. La descripción incluye el trabajo realizado para obtener el valor del error de deriva del eje $\mathrm{Y}$, el error de perpendicularidad entre los ejes $\mathrm{X}$ y $\mathrm{Y}$, así como la obtención del plano a partir de la caracterización de 56 líneas. El plano caracterizado fue empleado para construir una tabla de corrección tal que la incertidumbre de medición en el eje Z se redujo a menos de 15 micrómetros para cualquier punto medido dentro de una área de 4X5 metros. Adicionalmente, se describe también un método novedoso para medir el error en el ángulo de desviación de un pentaprisma y que hace uso de la MMC.

KEYWORDS: Prisms, Metrology, Geometric Optics.

\section{INTRODUCTION}

Flat surfaces of large size such as surface plates and tables are of considerable importance in precision engineering because they act as reference surfaces for the inspection of other workpieces. Assessment of the flatness of a surface can be accomplished in a variety of ways and with good fidelity. The simplest way is to employ a straightedge and observe where and how much light passes through the gap between edge and plate [1]. Other methods of measuring flatness include the use of a CMM [2], autocollimators [3] and precision levels [4]. Another important technique with high precision is that of interferometry, which enables the contour 
of the surface to be easily evaluated with a precision of $\lambda / 10$ [5]. However, some of the previous methods present serious difficulties when they are required to measure flats of large dimensions, i.e. of a few meters. In order to overcome the problems, new methods have been developed [6\&7].

In the following sections the characterization of a horizontal plane, within the measuring volume of a CMM of large dimensions, is described with the purpose of constructing a correction table in order to increase the performance of the machine.

\section{DESCRIPTION OF THE PROBLEM}

INAOE has constructed a CMM capable of measuring within a volume of 6X5X4 meters (see Fig. 1). Its first task will be to measure 192 near-plane surfaces of 3X5 meters with an uncertainty of measurement less than 15 micrometers. In principle, there are two strategies [8] employed to improve the accuracy of a CMM:

- Minimizing mechanical errors through design and precision manufacturing of the mechanical CMM structure.

- Software error compensation to correct systematic geometric errors and the errors induced by changes of temperature.



(a)

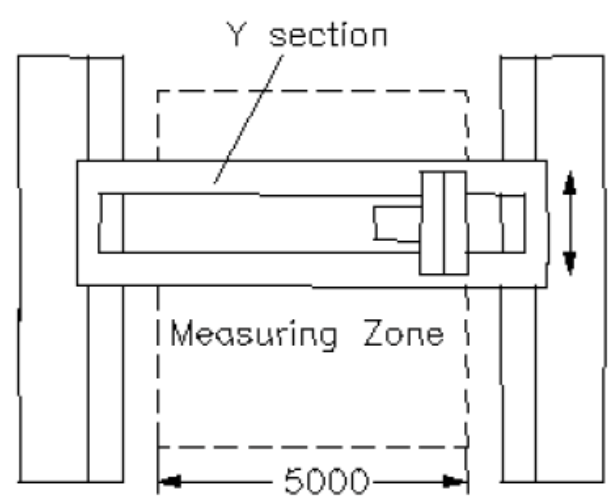

(b)

Figure. 1. Scheme of the CMM, a) front view, b) aerial view

Due to the dimensions of the machine it may be very expensive to improve its efficiency by applying the first method, and in our case we have concentrated on the second one. In a first attempt to define and reduce measurement errors we decided to characterize a horizontal plane within the measuring volume, with similar dimensions to the surfaces to be measured. With the obtained data we can then construct a correction table that allows us to reach the desired uncertainty.

The characterization of a plane in a small CMM can be made by employing instruments such as autocollimators and interferometers, which are placed on the table. The characterization is carried out along 8 lines and these are fitted in order to form a Union Jack pattern [1]. The INAOE CMM does not employ a surface plate, and the desired measurement plane is at a height of $2.40 \mathrm{~m}$, so it is difficult to use the traditional methods of measurement. In addition, the major measurement axis has a longitude of 5 meters and the desired separation between calibration lines is 100 millimeters, therefore, the measurement process must be repeated 51 times. To this number we must add three perpendicular lines and two diagonals (see Fig. 2) giving a total of 56 lines. 


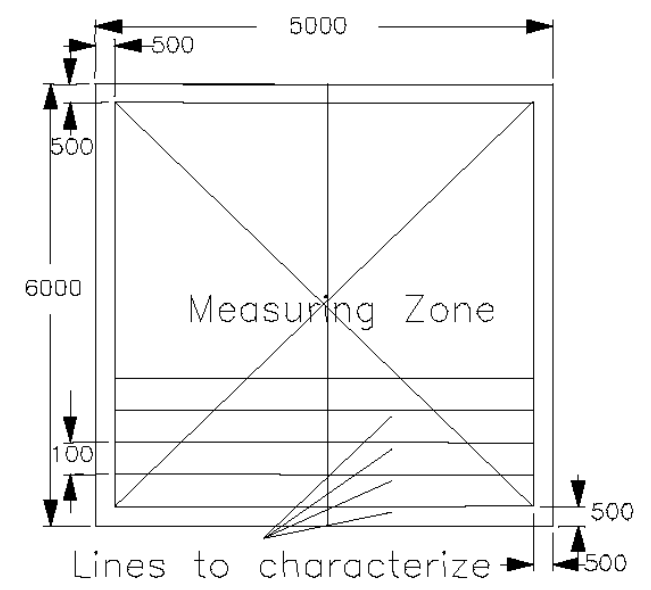

Figure. 2. Characterization of the lines

\section{THE UNION JACK PATTERN}

This consists in measuring a flat surface along eight lines, four along the edges, the diagonals and the centre lines (see Fig. 3a). In the traditional method, the datum plane is taken as passing through three of the corners, in Fig. $3 a A, B$ and $D$ are taken as having zero error. Thus, the datum table for each of the lines $A B, A D$ and $B D$ is adjusted to zero at the end. The significant point is now the centre of the diagonal BD. This value must obviously also be the error for this point on diagonal $\mathrm{AC}$ and the table for $\mathrm{AC}$ is therefore adjusted to give this value at the centre and zero at the end A. Next, the datum table for each of the lines DC and BC is adjusted to zero at the end $\mathrm{D}$ and $\mathrm{B}$, respectively, and the end corresponding at $\mathrm{C}$ is equal at the end of diagonal $\mathrm{AC}$ at the point $\mathrm{C}$. Lastly, the table for the line EG is adjusted so that the end at $\mathrm{E}$ must also be the error for this point on line AB and the end at G must be the error for this point on the line DC. Finally, the table for the line HF is adjusted in a similar way to the line EG.

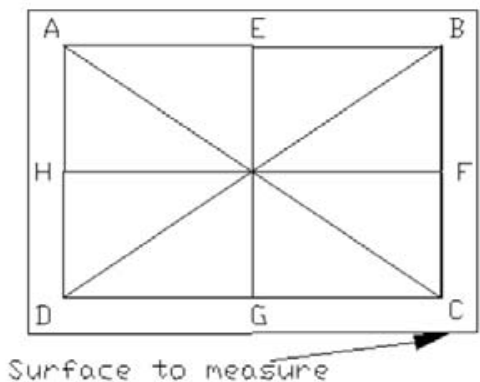

(a)

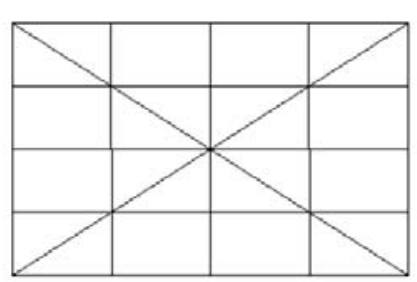

(b)

Fig. 3. Union Jack Pattern a) normal pattern, b) used pattern

For a more detailed survey, other generators parallel to the sides may be measured, splitting each side into four, eight, and so on.

In this case, we measure 51 lines in one direction and just 3 in the other direction, and the diagonals. The reference lines used in order to form the Union Jack pattern were four lines along the edges, two diagonals, two centre lines and four more semi-centre lines, two in one direction and two in the other (see Fig. 3b). The tables of the remaining lines were adjusted at the ends using the datum tables of the two lines of 5 meters. 


\section{CHARACTERIZATION OF THE PLANE}

\subsection{Obtaining the Y-axis yaw error}

The first step consisted on dividing the longest side of the measuring zone into five equal parts (see Fig. 4a). Subsequently, the straightness of the six lines was characterized using a collimated beam of approximately $4 \mathrm{~mm}$ diameter (formed with a $10 \mathrm{~mW}$ He-Ne laser and a collimator), a pentaprism and two quadrant detectors (QD) with a resolution of one micrometer. The collimated beam was adjusted parallel to the displacement of the Y-axis (see Fig.4b). The parallelism was carried out by placing a detector QD1 in the measuring head position of the Zaxis (see Fig 1) and displacing the Y-axis along its entire trajectory. The beam is aligned when the reading of QD1 at the beginning of the trajectory of the Y-axis is equal to the reading at the end. To maintain colinearity of the beam during the characterization of the six lines, another detector QD2 was placed in front of the laser. Any significant deviation of the beam from its initial path, after characterizing a line, was an indication to carry out an adjustment in the alignment of the parallelism and a repetition of the characterized line.

Next, a pentaprism was placed in the path of the beam to deviate it by approximately ninety degrees. QD1 was also rotated ninety degrees, so that it remained perpendicular to the deviated beam, and it was adjusted so that the initial reading was 0 in the $X$ direction of the detector (the initial position of the $X$-axis is shown in Fig. 1). Lastly, the $X$-axis of the machine was displaced a distance of 4 meters and other reading was taken. In this case, the exit beam is not parallel to the X-axis of the machine, so the reading at the end of the displacement is nonzero. This operation was repeated ten times for the six lines and an average of the final readings obtained for the $X$ direction of the detector are shown in Table I.

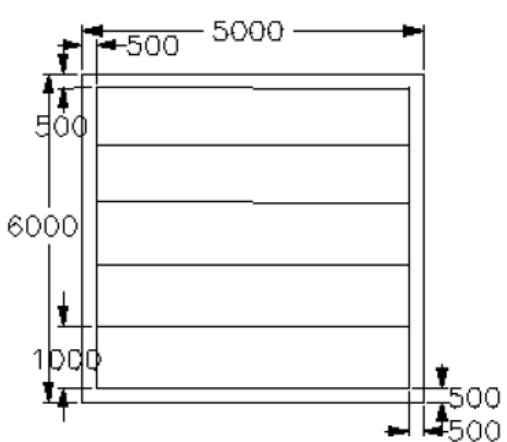

(a)

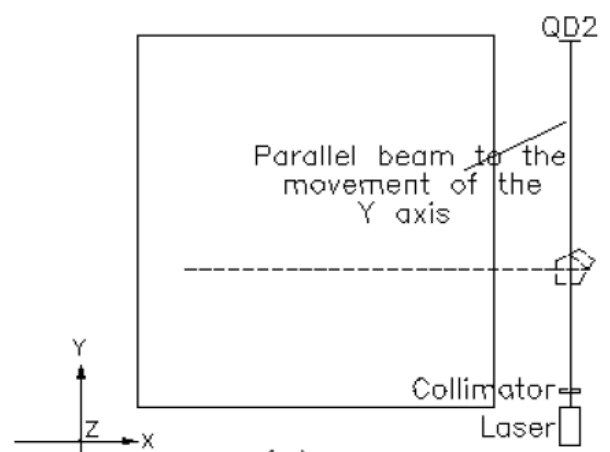

(b)

Figure. 4. Obtaining the yaw, a) division of the measuring zone b) location of the laser beam.

Table 1.

Readings obtained from the QD1

\begin{tabular}{|c|c|c|}
\hline Line Number & Initial reading & Final reading (X direction detector) $\mu \mathrm{m}$ \\
\hline 1 & 0 & -480 \\
\hline 2 & 0 & -567 \\
\hline 3 & 0 & -350 \\
\hline 4 & 0 & 351 \\
\hline 5 & 0 & -444 \\
\hline 6 & 0 & -618 \\
\hline
\end{tabular}


One can observe that the final readings are all different and this shows that the Y-axis exhibits yaw as it is displaced along its trajectory (in absence of yaw all readings must be equal and non-zero). The readings are within the interval $(-618,351)$ and therefore the total displacement is $0.969 \mathrm{~mm}$. Using the values $4000,0.969$ and carrying out some simple calculations it is easy to find that the Y-axis has a maximum yaw of 50 arcsec

\subsection{Obtaining the squareness error between the $X$ and $Y$ axes of the machine}

To obtain the squareness error it was necessary to measure the deviation angle of the pentaprism used to characterize the six lines. Two He-Ne lasers, two collimators, two diaphragms and three QDs were used for the measurement. Figure $5 \mathrm{a}$ shows the measurement setup. The laser L1 is the same source used previously to characterize the six lines and therefore it is considered to be pre-aligned. Two diaphragms, with a diameter of 4.2 millimeters, were placed in the path of the aligned beam. Laser L2 is then located, so that its beam also passes through the two diaphragms. Lastly, the parallelism of the beam was verified in the same manner as the laser L1, but in this case QD1 was rotated 180 degrees with respect to its initial position (see Fig. 1). In this manner, a beam traveling in both directions was generated.

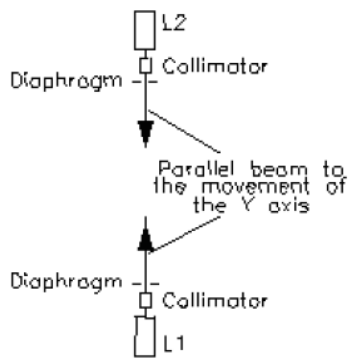

(a)
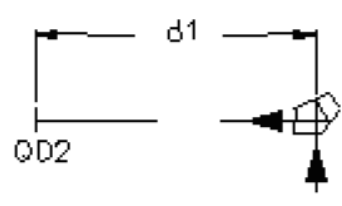

(b)

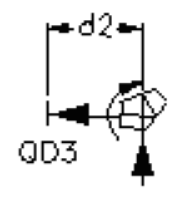

(c)

Figure 5. Method employed to measure the angle deviation error of the pentaprism a) construction of a beam traveling in both directions, b) position of QD2, c) placement of QD3

Following this set-up procedure the laser $\mathrm{L} 2$ is blocked with a card and the pentaprism is placed in the path of the unblocked beam (see Fig. 5b). The position of the exit beam is registered in QD2, placed at a distance d1 (2 meters). In this case the position of the beam on the detector is taken to be the zero measurement. Next, QD3 is placed at a distance $\mathrm{d} 2(0.2 \mathrm{~m})$ from the pentaprism and the reading of the beam position is taken (see Fig. $5 \mathrm{c}$ ). Finally, laser L1 is blocked, laser L2 is uncovered, the pentaprism is rotated ninety degrees and the position of the exit beam on QD3 is measured. After adjusting the pentaprism so that the beam coordinates coincide with the same point recorded previously (before rotation), QD3 is removed. Next, the reading of the position of the beam on QD2 (d3) is taken. This value is divided by two and the distance d4 (see Fig. 6a) is obtained. Using the distances $\mathrm{d} 1$ and $\mathrm{d} 4$ the error $(\mu)$ in the deviation angle of the pentaprism is obtained. The sign of the error depends on the direction in which the beam, after passing through the pentaprism, is displaced. For example, for the case shown in Fig. 6a the error is negative, while for the case of Fig. $6 \mathrm{~b}$ the sign is positive. The value obtained with this technique for the angle deviation error was -33 arcsec

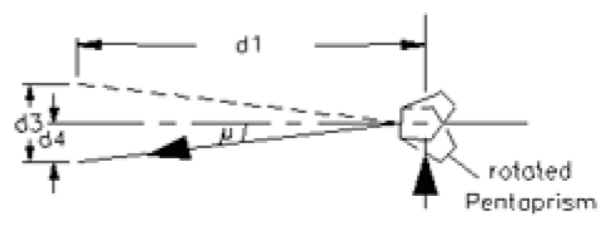

(o)

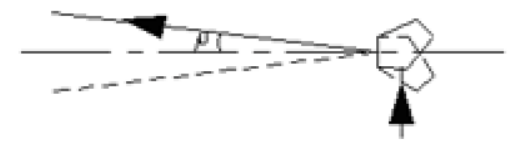

(b)

Figure 6. Determination of the sign of the angle deviation error a) case when sign is negative, b) case when sign is positive 
We then proceeded to obtain the squareness error between the $X$ and $Y$ axes. The displacement along of the $y$-axis, due to the total yaw, was 0.969 (see Fig. 7). This value is divided by 2 to obtain the middle point (-.1335) of the yaw. Using the values 4000 and -.1335 , the angle $\beta$ is obtained (6.88 arcsec). This angle is added to the angle deviation error $(\mu)$, giving the value 39.88 arcsec. Therefore, the midpoint value obtained to the squareness error between the $X$ and $Y$ axes was 39.9 arcsec. Figure 7 shows a graphical description of the process previously described.

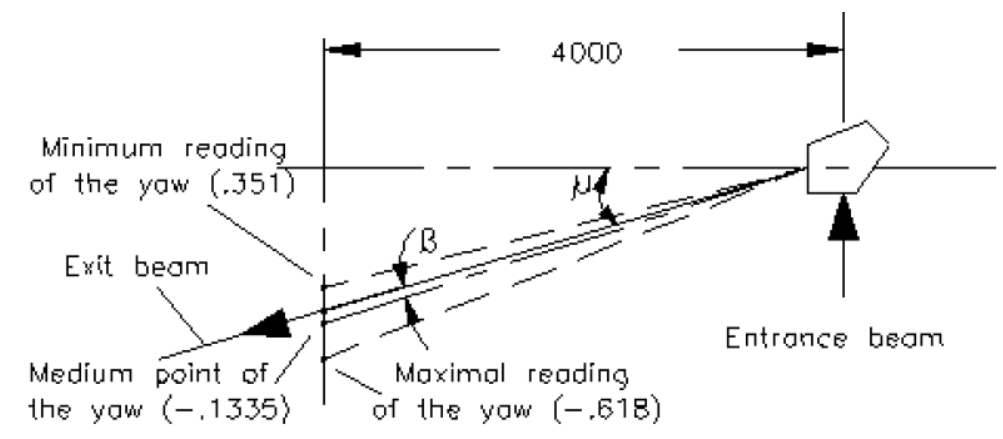

Figure 7. Graphic description

4.2.1 Error analysis for the measuring of the deviation angle

In above section $\mu=f(d 1, d 4)$, therefore the uncertainty of measurement of $\mu$ is given by

$$
\Delta \mu=\left|\frac{\partial f}{\partial d_{1}} \Delta d_{1}+\frac{\partial f}{\partial d_{4}} \Delta d_{4}\right| .
$$

where $\Delta \mathrm{d}_{1}$ and $\Delta \mathrm{d}_{4}$ are the uncertainty in the measurement of $\mathrm{d}_{1}$ and $\mathrm{d}_{4}$ respectively.

Differentiating Eq. (1), we obtain

$$
\Delta \mu=\left|\frac{d_{4}}{d_{1}^{2}} \Delta d_{1}\right|+\left|\frac{1}{d_{1}} \Delta d_{4}\right| .
$$

In the experiment distance $d_{1}$ is very large when compared to $d_{4}$, therefore the first term of the preceding equation can be neglected. In our experiment, $\Delta \mathrm{d}_{1}=0.5 \mathrm{~mm}, \Delta \mathrm{d}_{4}=.001 \mathrm{~mm}, \mathrm{~d}_{1}=2000 \mathrm{~mm}$ and $\mathrm{d}_{4}=.321 \mathrm{~mm}$. Using these values in Eq. (2) we obtain $\Delta \mu=.1$ arcsec

\subsection{Characterization of the lines}

Fig. 8a shows the arrangement employed in order to carry out the characterization of the lines. A laser beam is made parallel to the movement of the machine along the Y-axis, and is deviated by a pentaprism such that it travels cuasi- parallel (due to the discrepancy between squareness error of the CMM and the deviation angle error of the pentaprism) to the movement of the X-axis of the machine. After passing through the pentaprism, the exit beam is aligned only in the $Z$ direction of the machine (the laser is always fixed during the characterization process), since the beam is naturally cuasi-parallel in the $X$ direction. The $X$-axis of the machine travels automatically ten cycles with a speed of $40 \mathrm{~mm} / \mathrm{sec}$, and takes 200 seconds per cycle. The perpendicular displacements of the Z-axis of the machine with respect to the beam are registered, using a QD1 (rotated 90 degrees of its initial position, see Fig. 1) placed on the CMM measuring head, and the data are saved in a PC for 
posterior analysis. The time required to characterize a line was 35 minutes. Fig. 9 shows ten repeated measurements (scan paths) of the X-axis for the same line. For this case, the standard deviation on each point of maximum dispersion was estimated (see Table II). This operation was repeated for all 56 lines, and the standard deviation was shown not to exceed 6.5 micrometers.

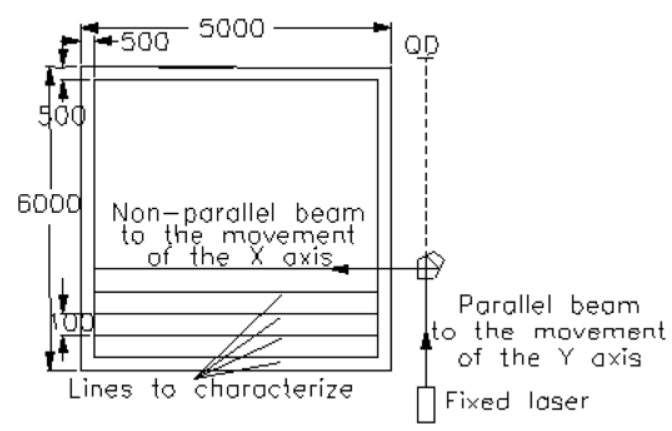

(a)

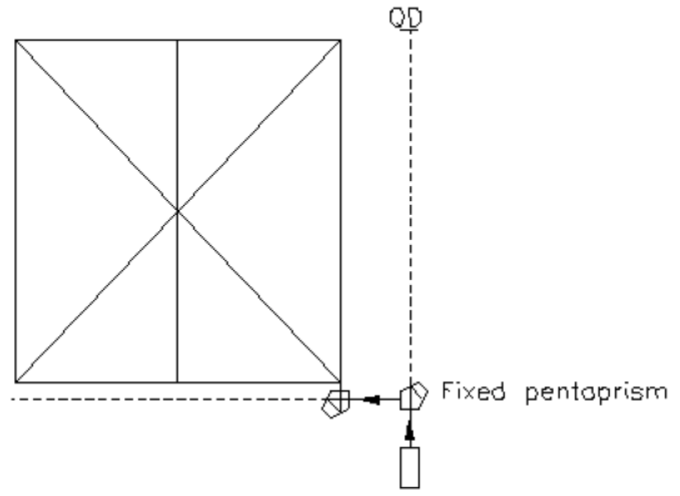

(b)

Figure 8. Characterization of the plane in order to construct the correction table a) measurement of the parallel lines to the X-axis of the machine

b) characterization of the three parallel lines to the $Y$ axis

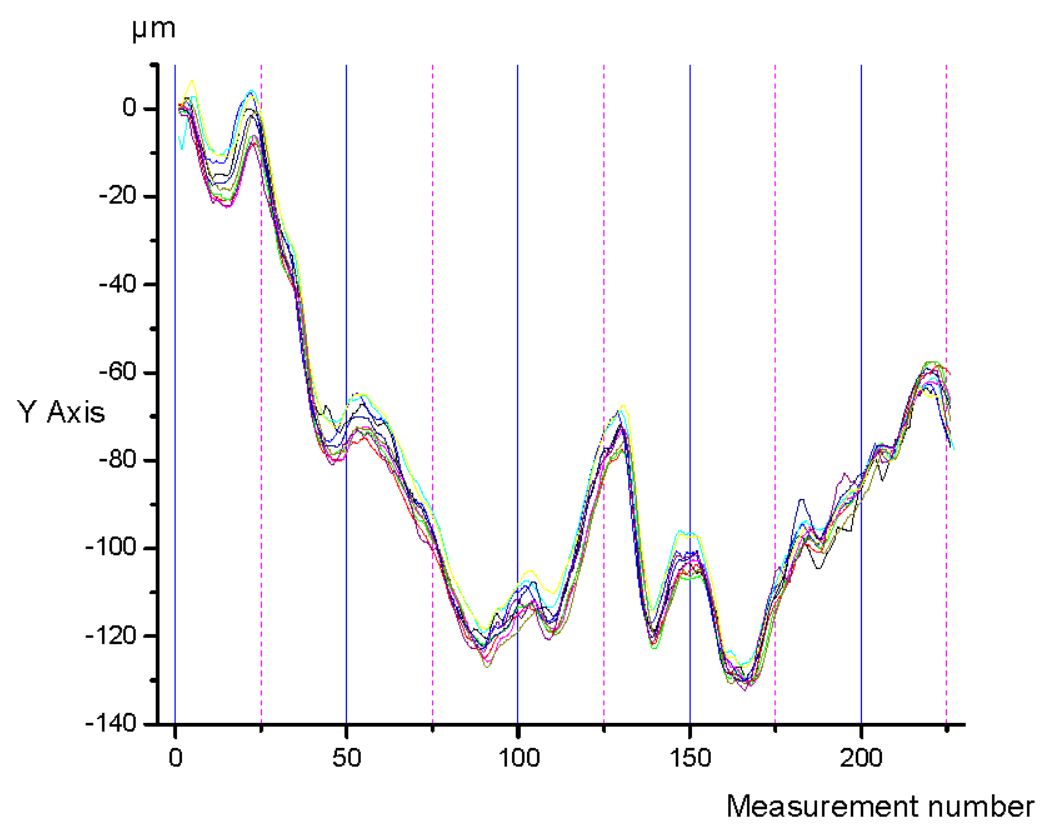

Figure 9. Curves of characterization of one line

After concluding the characterization of the set of X-axis lines, the three perpendicular lines were recorded. The pentaprism was placed outside the measuring area (see Fig. 8b) and another pentaprism was used to rotate the incident beam again, such that it traveled cuasi-parallel to the movement of the machine along the Y-axis. To characterize the diagonals the laser beam is aligned collinear with the diagonal movement of the measuring head. A three-dimensional graph of the characterized lines after adjustment following the Union Jack method is shown in Fig. 10. With this technique a flatness error of 98 micrometers was obtained (see vertical scale in Fig. 10). The error correction table uses the same data shown in Fig. 10 but with the sign inverted. 


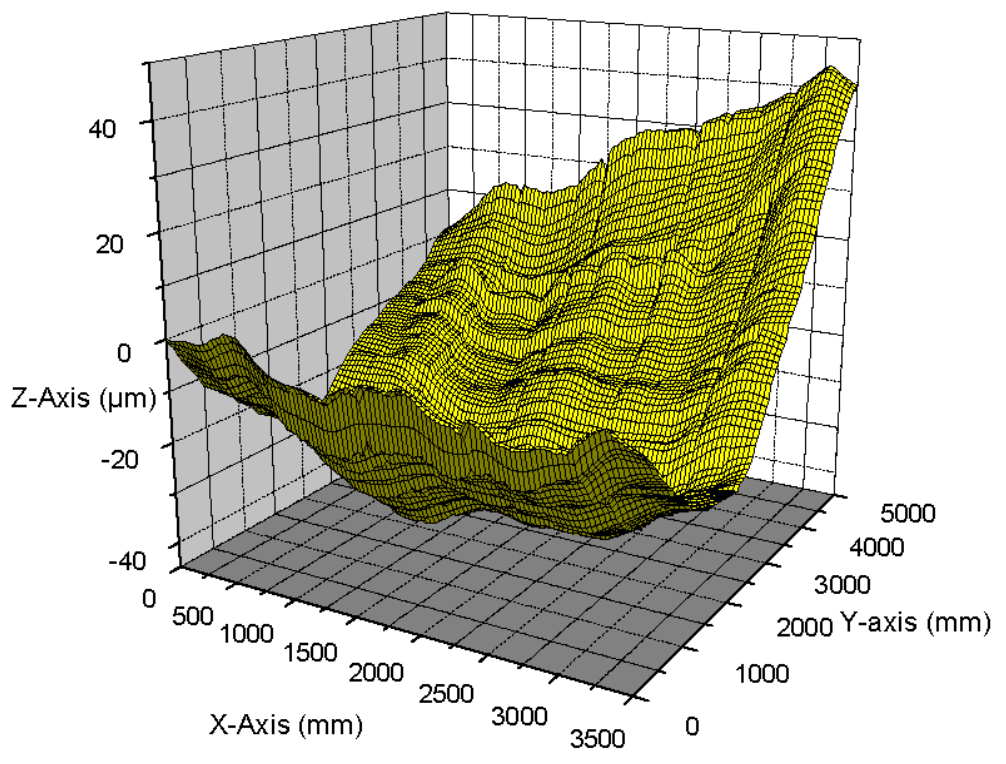

Figure 10. Three-dimensional view of the plane

\section{ERROR ANALYSIS FOR ADJUSTING THE UNION JACK PATTERN}

In the analysis a maximum standard measurement uncertainty of 5.4 micrometers is used for the end of the lines employed to form the Union Jack pattern (see Table II).

Table II.

Standard deviation obtained on points of maximum dispersion of the Figure 9

\begin{tabular}{|c|c|c|c|c|c|c|c|c|c|c|c|c|}
\hline No. of point & Line 1 & Line 2 & Line 3 & Line 4 & Line 5 & Line 6 & Line 7 & Line 8 & Line 9 & Line 10 & Average & Stan. Devi. (S) \\
\hline 1 & 1 & 1 & -1 & 0 & 6 & 0 & 0 & 0 & 0 & -1 & -1 & 2 \\
\hline 18 & -8 & -19 & -17 & -2 & -5 & -20 & -7 & -15 & -12 & -18 & -12 & 6.4 \\
\hline 22 & 0 & -9 & -6 & 4 & 4 & -8 & 3 & -2 & -2 & -8 & -2 & 5.5 \\
\hline 55 & -67 & -75 & -74 & -67 & -65 & -72 & -65 & -73 & -70 & -75 & -70 & 4.1 \\
\hline 100 & -115 & -115 & -113 & -110 & -109 & -115 & -108 & -119 & -113 & -112 & -113 & 3.3 \\
\hline 150 & -104 & -105 & -107 & -101 & -96 & -103 & -97 & -106 & -102 & -102 & -102 & 3.6 \\
\hline 226 & -77 & -60 & -67 & -76 & -75 & -66 & -75 & -71 & -68 & -74 & -71 & 5.4 \\
\hline
\end{tabular}

To form the pattern 8 lines are employed (see Fig. 3), however, we begin with a single line, for example, the line $A B$ (initial line) and whenever new lines (lines $A D$ and $A C$ ) are joined to the end of the initial line their uncertainties are added to the uncertainty of the initial line. When some other lines are incorporated, their uncertainties are added and so on until the complete pattern is formed. The uncertainty of each line is shown in Fig. 11, where we can see that they are not equal for all lines. The maximum uncertainty is at point $C$ and the minimum is along the initial line.

When all of the lines are joined, the maximum uncertainty is increased up to seven times the value 5.4 and the minimum to two times that same value. According to the laws of probability, when quantities are added, each containing an error S, S2, or any similar accidental type, their uncertainty can be calculated with equation [9]: 


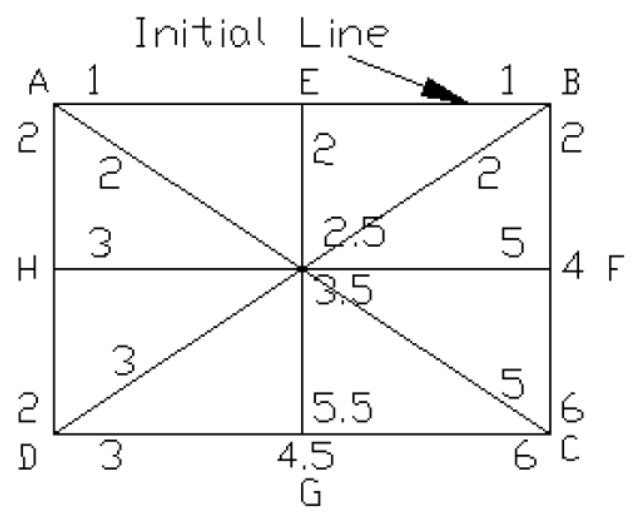

Figure 11. Distribution of accumulative uncertainties on the Union Jack Pattern

$$
S_{\text {sum }}=\sqrt{S_{1}^{2}+S_{2}^{2}+\ldots . .+S_{7}^{2}}
$$

obtaining a maximum standard measurement uncertainty of 14.3 micrometers and a minimum of 7.6.

\section{COMMENTS}

In the construction of the co-linear beams employed for the characterization of the pentaprism (section 3.2), it is more important that they are parallel, rather than that they are co-linear. This is because an error in the parallelism contributes more to the error in the measurement than an error in the co-linearity. Parallelism is improved by using large distances between the lasers, of the order of 4 or 5 meters, and shorter distances between the pentaprism and QD3. A good distance would be such that the QD3 could resolve the displacements of the two impinging beams. An inconvenience of having large distances is that fluctuations in the environment perturb the path of the laser and therefore installations with a carefully controlled environment are required. This is an important disadvantage of the method employed. During our measurements a maximum beam wobble of 10 micrometers over a distance of 5 meters was observed. This contributed 0.5 arcsec of error in the measurement of the deviation angle. Another error that must be considered is due to the parallelism between the beams. In this case the error in the parallelism was less than $0.020 \mathrm{~mm}$, which resulted in an angular error of the order of 0.8 arcsec.

\section{CONCLUSIONS}

A technique has been described for characterizing a measurement plane within a CMM of large dimensions without using instruments such as autocollimators and interferometers. The characterization differed from the traditional methods since it was not possible to use these methods for this type of machine construction, principally due to the dimensions involved. For the Y-axis Yaw a value of 50 arcsec was obtained and for the squareness error between the $X$ and $Y$ axes a value of -39.9 arcsec was recorded. The uncertainty of measurement for both values was below 1.5 arcsec.

Taking into account that the standard uncertainty at the end of all of the characterized lines were within 5.4 micrometers, we find a variable measurement standard uncertainty less than 15 micrometers, applied solely to the Z- axis, for any measurement taken within the characterized measuring area after application of the correction table. This allows us to achieve a precision within the requested 15 micrometers. 
Lastly, the uncertainty in the deviation angle measurement was less than 1 arcsec therefore we consider it is an excellent method to measure other components having right angles, like dove prisms, although we realize that it is a very expensive technique.

\section{References}

[1] Whitehouse, D., Handbook of Surface Metrology, Inst. of physics publishing, London, pp. 120-126, 1994.

[2] International Standard ISO 230-1:1996(E), Test code for machine tools - Part I, pp. 22, 1996.

[3] Young, A. W. W., Optical Workshop instruments, Chap. 7 in Applied Optics and Optical Engineering, Vol. IV, Rudolph Kingslake, Ed., Academic Press, New York, pp. 221-223, 1967.

[4] Horne, D. F., Optical Instruments and Their Applications, Adam Hilger Ltd., London, pp. 234-237, 1980.

[5] Malacara, D, Newton, Fizeau, and Haidinger interferometers, Chap. 1 in Optical Shop Testing, Malacara, D. Ed., John Wiley and Sons, New York, pp. 1-12, 1978.

[6] Aketagawa M., Takada K. and Kon H., Calibration of a laser reference plane for large-scale dimensional flatness measurement, Meas. Sci. Technol. Vol. 9, 1115-1119, 1998.

[7] Aketagawa M., Takada K., Torii K., Kozakura Y. and Hirano Y., Laser reference plane for flatness measurement of large-scale machined surfaces, Int. J. Japan Soc. Precision Eng., 28, 261-266, 1994.

[8] Bosch J., Coordinate Measuring Machines and Systems, Marcel Dekker, Inc., New York, pp. 279, 1995

[9] Austin B. B., Errors in Practical Measurement in Science, Engineering and Technology, John Wiley and Sons, Inc, New York, pp. 72, 1985. 